\title{
DEFORMIDADE DE SPRENGEL: TRATAMENTO CIRÚRGICO PELA TÉCNICA DE GREEN MODIFICADA
}

\author{
SPRENGEL'S DEFORMITY: SURGICAL \\ CORRECTION BY A MODIFIED GREEN PROCEDURE
}

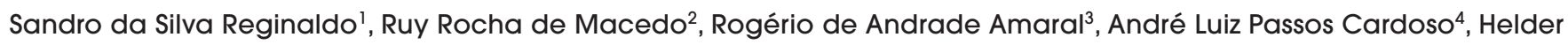
Rocha Silva Araújo ${ }^{4}$, Sergio Daher ${ }^{5}$

\section{RESUMO}

Objetivo: Demonstrar os resultados estéticos e funcionais de pacientes submetidos à correção cirúrgica de escápula alta congênita - deformidade de Sprengel - por uma modificação da técnica de Green, bem como avaliar o grau de satisfação dos pacientes e as complicações da técnica utilizada. Métodos: Foram avaliados nove pacientes operados pela técnica de Green modificada, no período de setembro de 1993 a abril de 2008 . Como modificação da técnica original foram realizados descolamento muscular subperiosteal, ressecção apenas da porção súpero-medial da escápula e, em vez da utilização de tração esquelética, optou-se pela fixação com fio de aço subcutâneo da porção medial da espinha da escápula à crista ilíaca posterior contralateral. A idade média dos pacientes foi de sete anos e três meses. O seguimento pós-operatório médio foi de três anos e sete meses. Resultados: Houve incremento médio na elevação de cerca de $39^{\circ}$ (variando de $0^{\circ}$ a $80^{\circ}$ ). Segundo a classificação de Cavendish, obteve-se a melhora estética de dois graus em oito casos e de três graus em um. Todos os pacientes ficaram satisfeitos com o resultado. Conclusões: Os pacientes com deformidade de Sprengel submetidos a tratamento cirúrgico por meio de uma modificação da técnica de Green, com fixação da escápula na crista ilíaca posterior contralateral em vez de se utilizar tração esquelética, apresentaram melhora tanto funcional como estética; todos os pacientes e/ou familiares ficaram satisfeitos e as complicações relacionadas com a técnica cirúrgica não interferiram no resultado final.

\section{ABSTRACT}

Objective: To evaluate the cosmetic and functional results of patients submitted to surgical correction of Congenital High Scapula (Sprengel's Deformity) using modified Green's Procedure, as well as patients' satisfaction and complications. Methods: Nine patients submitted to surgical treatment from September 1993 to April 2008 have been assessed. The modification from original technique was: subperiosteal muscle detachment, resection of superomedial scapular portion and fixation of medial portion of scapular spine to contralateral posterior iliac crest instead of skeletal traction, with subcutaneous wire. The mean age was 7 years and 3 months. The mean follow-up time was 3 years and 7 months. Results: The mean improvement in forward elevation was 39o (range 0 to 80o). According to the Cavendish Classification, cosmetic improvement of two degrees was achieved in eight cases, and three degrees in one. All patients were satisfied with results. Conclusions: Surgical correction of Sprengel's Deformity by a modified Green's procedure with contralateral posterior iliac crest fixation instead of skeletal traction, showed both cosmetic and functional improvements; all patients and/or family members were satisfied with the results, and the complications associated to the surgical technique did not interfere on end results.

Keywords - Sprengel's deformity; Scapula

Descritores - Deformidade de Sprengel; Escápula

\footnotetext{
1 - Chefe do Grupo de Ombro e Cotovelo do Hospital das Clínicas da Faculdade de Medicina da Universidade Federal de Goiás.

2 - Médico Assistente do Grupo de Ombro e Cotovelo do Hospital das Clínicas da Faculdade de Medicina da Universidade Federal de Goiás.

3 - Ex-estagiário do Grupo de Ombro e Cotovelo do Hospital das Clínicas da Faculdade de Medicina da Universidade Federal de Goiás.

4 - Médico Assistente do Departamento de Ortopedia e Traumatologia da Faculdade de Medicina da Universidade Federal de Goiás.

5 - Professor do Departamento de Ortopedia e Traumatologia da Faculdade de Medicina da Universidade Federal de Goiás.
} 


\section{INTRODUÇÃO}

A elevação congênita da escápula é uma condição rara e de etiologia desconhecida que resulta da interrupção anormal da migração caudal da escápula no período embrionário ${ }^{(1-4)}$. Eulenberg fez a primeira descrição em 1863 e, a partir da publicação do trabalho de Kolliker, em 1891, creditando a Sprengel esse achado, passou-se a utilizar o termo deformidade de Sprengel ${ }^{(1,2,5,6)}$.

As alterações clínicas principais são o posicionamento anormal e a hipoplasia da escápula, podendo acarretar não só problemas estéticos como também limitação dos movimentos da cintura escapular ${ }^{(1,2,7,8)}$ (Figura 1). Chinn relata a possibilidade de o diagnóstico ser feito ainda no período pré-natal por meio de ultrassonografia ${ }^{(9)}$.

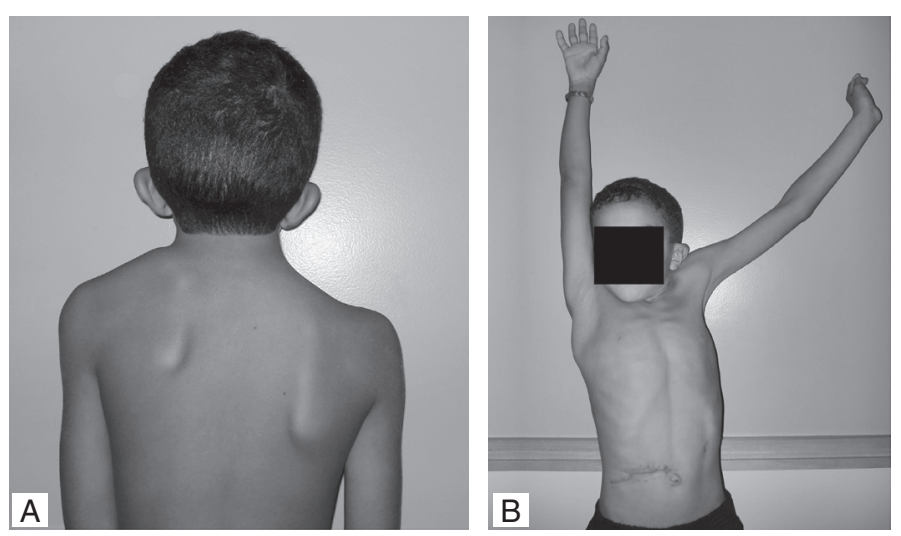

Figura 1 - Alterações clínicas na Deformidade de Sprengel. Escápula esquerda hipoplásica e elevada (a) e limitação da elevação (b) (caso 9).

A presença do osso omovertebral, que une a escápula à coluna vertebral, é uma alteração característica citada por vários autores ${ }^{(1,7,10-12)}$. Mooney et al relatam um caso em que foi identificada uma estrutura óssea que se estendia da borda medial da escápula em direção à clavícula e à região occipital, diferente do padrão habitual do osso omovertebral ${ }^{(13)}$.

$\mathrm{O}$ tratamento cirúrgico da deformidade visa à melhora estética e, em alguns casos, a funcional, podendo consistir de osteotomias, ressecções ósseas, liberações musculares com reposicionamento da escápula ou uma associação destas ${ }^{(4,6,8,10,11,14-16)}$. A técnica cirúrgica descrita por Green em 1957 é uma das mais utilizadas e envolve ressecção óssea da porção supraclavicular da escápula, além do rebaixamento da mesma, que é mantido por meio de tração esquelética ${ }^{(17)}$. Leibovic et al ${ }^{(4)}$ descrevem uma modificação dessa técnica, em que a escápula é mantida rebaixada por meio de uma sutura com uma bolsa feita no músculo grande dorsal, não sendo necessária a tração esquelética. Aydinli et al ${ }^{(18)}$ acrescentam a essa modificação a osteotomia da clavícula como primeiro tempo cirúrgico e a fixação do polo inferior da escápula a uma costela adjacente por meio de sutura absorvível. Monterrubio e Cázares ${ }^{(19)}$ propõem como modificação à técnica original de Green a osteotomia da base do processo coracoide e a fixação da escápula a uma costela inferior com sutura absorvível.

O objetivo deste trabalho é demonstrar os resultados estéticos e funcionais de pacientes submetidos à correção cirúrgica de escápula alta congênita - deformidade de Sprengel - por uma modificação da técnica de Green, bem como avaliar o grau de satisfação dos pacientes e/ou familiares e as complicações da técnica utilizada.

\section{MÉTODOS}

No período de setembro de 1993 a abril de 2008, foram tratados, pelo Grupo de Ombro e Cotovelo do Hospital das Clínicas da Faculdade de Medicina da Universidade Federal de Goiás, 14 pacientes com diagnóstico de deformidade de Sprengel, porém cinco não tiveram acompanhamento mínimo de seis meses, restando nove pacientes, que são o objeto deste estudo.

A idade dos pacientes variou de quatro anos e 10 meses a 10 anos (média de sete anos e três meses). Sete eram do sexo masculino e dois, do feminino. Em relação ao ombro acometido, oito eram do lado esquerdo e um, do direito.

Dentre os casos avaliados, apenas dois pacientes não apresentavam deformidades associadas. Entre as deformidades encontradas, as mais freqüentes foram: KlippelFeil (quatro casos), escoliose (três casos) e deformidades de costelas (três casos). Também observamos presença de lábio leporino, hérnia umbilical, dedo em gatilho e malformação renal. Em apenas um paciente (caso 6) foi diagnosticada presença de osso omovertebral (Figura 2).

Todos os pacientes foram submetidos a tratamento cirúrgico. Além dos aspectos estéticos, a indicação para tratamento cirúrgico também objetivou melhora funcional do ombro, principalmente por meio do ganho de elevação.

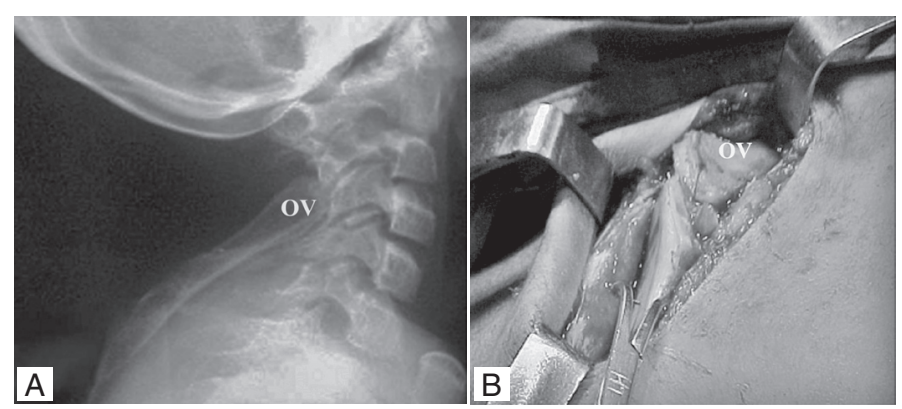

Figura 2 - Osso omovertebral (OV). Aspecto radiográfico (a) e intra-operatório (b) (caso 6). 
A avaliação clínica pré-operatória consistiu da mensuração da elevação do ombro acometido, que variou de $80^{\circ}$ a $160^{\circ}$ (média de $110^{\circ}$ ); a deformidade estética foi avaliada segundo os critérios de Cavendish ${ }^{(6)}$ (Tabela 1), sendo cinco pacientes do grau III e quatro do grau IV.

Tabela 1 - Escala de Cavendish para classificar esteticamente pacientes com deformidade de Sprengel ${ }^{6}$.

\begin{tabular}{l|l}
\hline $\begin{array}{l}\text { Grau I } \\
\text { (muito leve) }\end{array}$ & $\begin{array}{l}\text { Ombros nivelados e deformidade praticamente invisível quando } \\
\text { o paciente está vestido. }\end{array}$ \\
\hline $\begin{array}{l}\text { Grau II } \\
\text { (leve) }\end{array}$ & $\begin{array}{l}\text { Ombros praticamente nivelados, mas a deformidade é visível } \\
\text { com paciente vestido. }\end{array}$ \\
\hline $\begin{array}{l}\text { Grau III } \\
\text { (moderada) }\end{array}$ & Ombro elevado de 2 a $5 \mathrm{~cm}$ e a deformidade é facilmente visível. \\
\hline $\begin{array}{l}\text { Grau IV } \\
\text { (grave) }\end{array}$ & $\begin{array}{l}\text { Ombro bastante elevado, com ângulo superior da escápula } \\
\text { próximo do occipital. }\end{array}$ \\
\hline
\end{tabular}

A técnica cirúrgica utilizada foi uma modificação da descrita por Green, que originalmente consiste de um descolamento extraperiosteal dos músculos superiores e mediais da escápula, ressecção do osso omovertebral (se presente), excisão da porção supraclavicular da escápula, rebaixamento da escápula e reinserção da musculatura na nova posição, com manutenção desta posição com fio de tração esquelética ${ }^{(17)}$. Como modificação da técnica original foram realizados descolamento muscular subperiosteal, ressecção apenas da porção súpero-medial da escápula e, em vez da utilização de tração esquelética, optou-se pela fixação com fio de aço subcutâneo da porção medial da espinha da escápula à crista ilíaca posterior contralateral $^{(20)}$ (Figuras 3 e 4).

No pós-operatório, os pacientes permaneceram com imobilização gessada tipo Velpeau por cerca de quatro semanas. O material de fixação (fio de aço) foi retirado, em média, após 69 dias (variando de 30 a 110 dias) do procedimento cirúrgico inicial.

Para avaliação pós-operatória foram utilizados os mesmos critérios estéticos e funcionais do pré-operatório, acrescidos do grau de satisfação do paciente e/ou dos familiares (satisfeitos/insatisfeitos), do aspecto da cicatriz (normotrófica/hipertrófica) e da presença de complicações relacionadas com a técnica cirúrgica utilizada. Na avaliação radiográfica foi medido o rebaixamento da escápula como descrito por Carson et $a l^{(11)}$, ou seja, a diferença entre o desnivelamento escapular pós-operatório e o pré-operatório.

O seguimento pós-operatório médio foi de três anos e sete meses, variando de oito meses a sete anos e 11 meses.
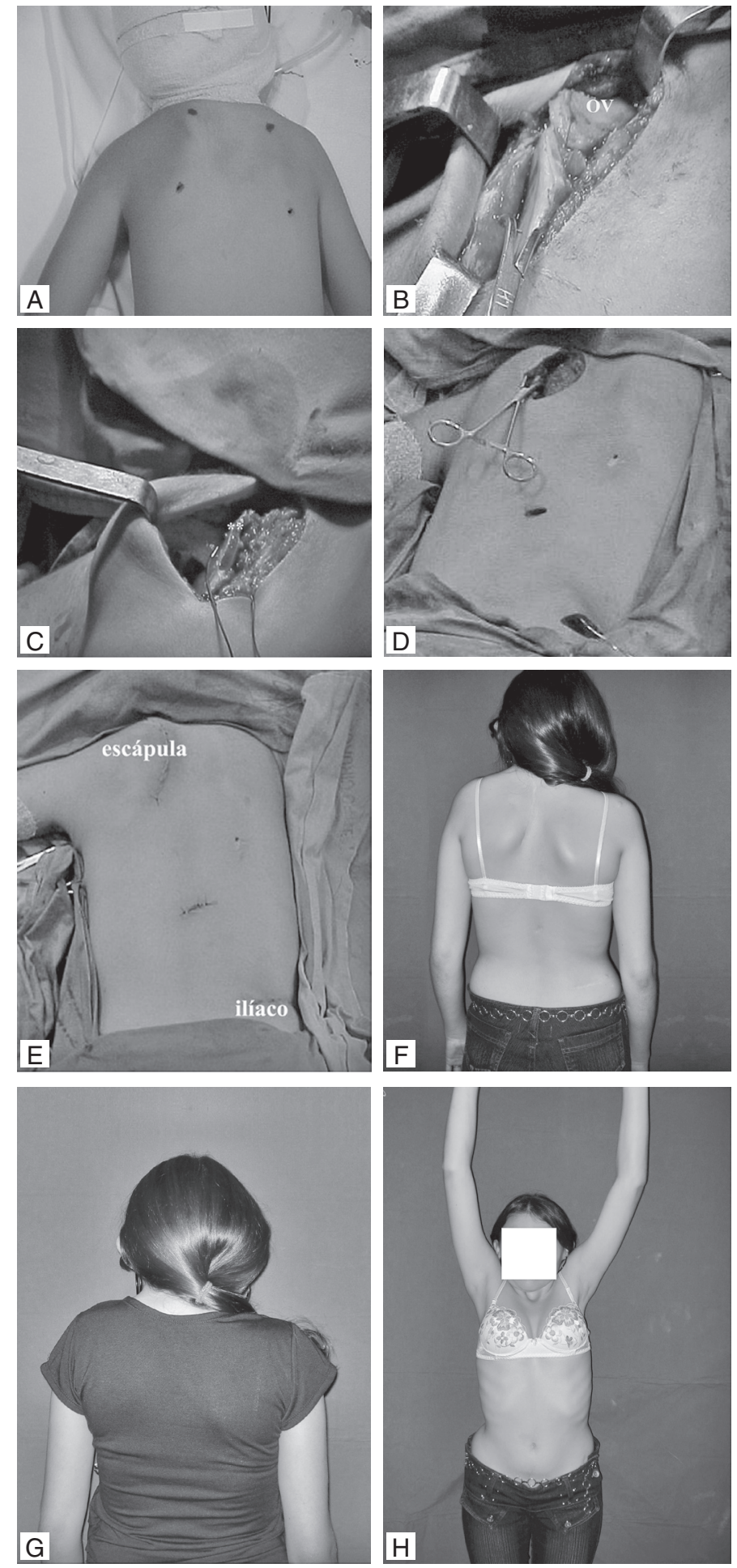

Figura 3* - Técnica de Green modificada (caso 6): a) paciente em decúbito ventral horizontal, com ângulos escapulares marcados (pontos pretos); b) desinserção subperiosteal da musculatura medial, rebaixamento da escápula e excisão do osso omovertebral (ov); c) porção súpero-medial da escápula ressecada $\left({ }^{\star *}\right)$ e fio de aço fixado à espinha da escápula; d) passagem subcutânea do fio de aço até a crista ilíaca posterior contralateral, onde será fixado para manutenção do rebaixamento escapular; e) aspecto cirúrgico final; f,g,h) aspecto clínico com 6 anos e 6 meses de seguimento pós-operatório, demonstrando melhora estética e da elevação.

* Retirado, com autorização do autor, de Lech O, Reginaldo SS. Deformidade de Sprengel (Escápula Alada). In: Lech O. Defeitos Congênitos nos Membros Superiores. 


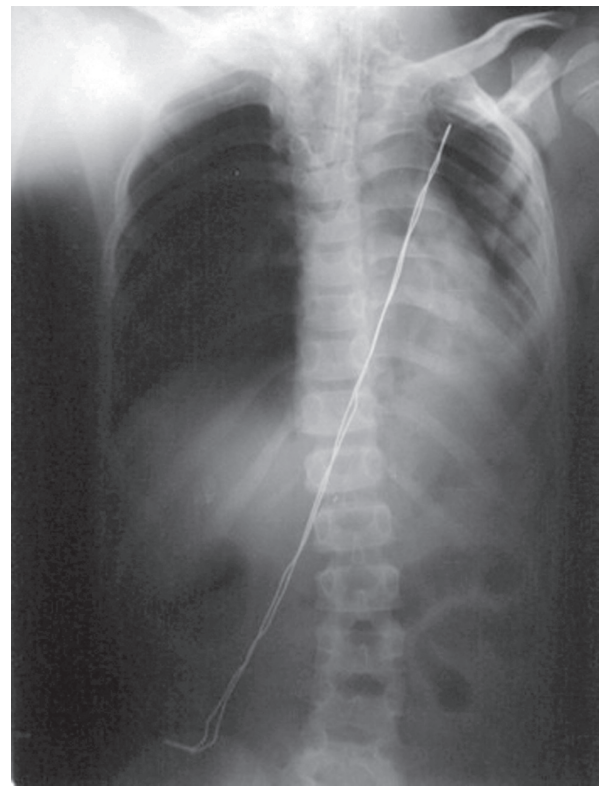

Figura 4 - Aspecto radiográfico no pós-operatório imediato (caso 8).

\section{RESULTADOS}

Houve incremento médio na elevação de cerca de $39^{\circ}$ (variando de $0^{\circ}$ a $80^{\circ}$ ); a variação da elevação no pósoperatório foi de $130^{\circ}$ a $160^{\circ}$, com média de $149^{\circ}$. Segundo a classificação de Cavendish, obteve-se melhora de dois graus em oito casos e de três graus em um; seis pacientes passaram para grau I e três para grau II.

O rebaixamento radiográfico médio foi de $2,7 \mathrm{~cm}$ (variando de 1 a $5,8 \mathrm{~cm}$ ).

Quanto às cicatrizes, todas foram consideradas normotróficas.

Todos os nove pacientes e/ou seus familiares ficaram satisfeitos com os resultados.

Dentre as complicações pós-operatórias, houve quebra do material de fixação em três casos e infecção superficial em um caso. Essas complicações não interferiram diretamente nos resultados finais.

A tabela 2 contém um resumo da casuística e resultados.

Tabela 2 - Dados dos pacientes com Deformidade de Sprengel operados pela técnica de Green modificada

\begin{tabular}{|c|c|c|c|c|c|c|c|c|c|}
\hline & Caso1 & Caso 2 & Caso 3 & Caso 4 & Caso 5 & Caso 6 & Caso 7 & Caso 8 & Caso 9 \\
\hline Iniciais & MAGJ & LHPO & GPS & EDMB & FSC & TMS & GCV & DSL & CDSP \\
\hline Sexo & M & $M$ & M & M & M & $F$ & $\mathrm{~F}$ & M & M \\
\hline Lado & E & D & $E$ & E & $E$ & E & $E$ & E & $E$ \\
\hline Idade & $7 a 2 m$ & $5 a 4 m$ & $8 a 11 \mathrm{~m}$ & $4 a \mathrm{a} 10 \mathrm{~m}$ & $6 a 3 m$ & $6 a 9 m$ & $9 a 2 m$ & $10 a$ & $7 a 5 m$ \\
\hline $\begin{array}{l}\text { Deformidades } \\
\text { associadas }\end{array}$ & $\begin{array}{l}\text { Escoliose } \\
\text { Ausência de } \\
\text { costela E }\end{array}$ & Não & $\begin{array}{l}\text { Escoliose } \\
\text { Klippel-Feil }\end{array}$ & $\begin{array}{l}\text { Hipoplasia de } \\
\text { costela }\end{array}$ & $\begin{array}{l}\text { Klippel-Feil } \\
\text { Deformidade de } \\
\text { costela } \\
\text { Má-formação renal }\end{array}$ & $\begin{array}{l}\text { Escoliose } \\
\text { Lábio leporino } \\
\text { Hérnia umbilical } \\
\text { Osso omovertebral } \\
\text { Má-formação renal }\end{array}$ & $\begin{array}{l}\text { Klippel-Feil } \\
\text { Hemi-vértebra C6 }\end{array}$ & Klippel-Feil & Não \\
\hline Cirurgia & 03/09/1993 & 25/06/1996 & 17/06/1998 & 02/06/1999 & 29/11/1999 & 28/03/2001 & 23/05/2002 & $16 / 04 / 2003$ & $21 / 05 / 2007$ \\
\hline Retirada do fio & 30 dias & 80 dias & 37 dias & 64 dias & 68 dias & 70 dias & 75 dias & 110 dias & 87 dias \\
\hline $\begin{array}{l}\text { Cavendish } \\
\text { pré-operatório }\end{array}$ & III & III & IV & III & IV & IV & III & IV & III \\
\hline $\begin{array}{l}\text { Cavendish } \\
\text { pós-operatório }\end{array}$ & 1 & 1 & II & I & I & II & I & II & 1 \\
\hline $\begin{array}{l}\text { Elevação } \\
\text { pré-operatória }\end{array}$ & $110^{\circ}$ & $90^{\circ}$ & $110^{\circ}$ & $160^{\circ}$ & $80^{\circ}$ & $80^{\circ}$ & $160^{\circ}$ & $90^{\circ}$ & $110^{\circ}$ \\
\hline $\begin{array}{l}\text { Elevação } \\
\text { pós-operatória }\end{array}$ & $150^{\circ}$ & $130^{\circ}$ & $140^{\circ}$ & $160^{\circ}$ & $130^{\circ}$ & $160^{\circ}$ & $160^{\circ}$ & $150^{\circ}$ & $160^{\circ}$ \\
\hline $\begin{array}{l}\text { Rebaixamento } \\
\text { da escápula }\end{array}$ & $1 \mathrm{~cm}$ & $1,6 \mathrm{~cm}$ & $3,3 \mathrm{~cm}$ & $1,8 \mathrm{~cm}$ & $5,8 \mathrm{~cm}$ & $2,1 \mathrm{~cm}$ & $2,5 \mathrm{~cm}$ & $2,4 \mathrm{~cm}$ & $3,3 \mathrm{~cm}$ \\
\hline Complicações & Não & $\begin{array}{l}\text { Infecção } \\
\text { superficial }\end{array}$ & Não & Quebra do fio & Não & Quebra do fio & Quebra do fio & Não & Não \\
\hline Satisfação & Satisfeito & Satisfeito & Satisfeito & Satisfeito & Satisfeito & Satisfeita & Satisfeita & Satisfeito & Satisfeito \\
\hline $\begin{array}{l}\text { Seguimento } \\
\text { pós-operatório }\end{array}$ & 7a $11 \mathrm{~m}$ & $4 a 6 m$ & $3 a$ & $2 a 2 m$ & 1a $9 m$ & $6 a 6 m$ & $5 a 4 m$ & $8 m$ & $11 \mathrm{~m}$ \\
\hline
\end{tabular}




\section{DISCUSSÃO}

A deformidade de Sprengel é a deformidade congênita mais comum no ombro ${ }^{(3,10)}$. Resulta de um bloqueio da descida normal da escápula, provavelmente entre a nona e $12^{\mathrm{a}}$ semanas de gestação, ocasionando um osso em posição anormal e usualmente hipoplásico, além de a musculatura periescapular apresentar graus variáveis de hipoplasia e alterações degenerativas ${ }^{(2,3,6,7,11)}$.

As deformidades esqueléticas associadas à deformidade de Sprengel são freqüentes, variando de $67 \%$ a $100 \%^{(1,3,4,16,21)}$. Sete de nossos casos $(78 \%)$ apresentaram deformidades associadas, sendo as mais freqüentes as da coluna vertebral (escoliose e Klippel-Feil) e as alterações de costelas.

Uma alteração considerada característica por muitos autores é a presença de uma conexão omovertebral que une o ângulo súpero-medial da escápula ao processo espinhoso, à lâmina ou ao processo transverso da coluna cervical baixa. Essa conexão pode ser de natureza fibrosa, cartilaginosa, óssea ou uma combinação dessas $^{(1,10,11)}$. Sartoris ${ }^{(22)}$ considera que a radiografia em incidência ântero-posterior ou a tomografia computadorizada com reconstrução em três dimensões são os melhores exames para o detecção do osso omovertebral. Os relatos da presença do osso omovertebral na literatura indicam incidência de $20 \%$ a $46 \%{ }^{(6,8,11,12,16)}$, porém, apenas um paciente de nossa série (11\%) apresentou essa conexão.

Em deformidades leves não está indicado tratamento cirúrgico, reservado para pacientes com deformidades mais acentuadas ${ }^{(1,2,6,14)}$. Segundo Cavendish ${ }^{(6)}$, o objetivo primário do tratamento é a melhora estética, já que a melhora da função não é consistente. Carson et al ${ }^{(11)}$ sugerem que a melhora da função pode ser também um objetivo nos casos com importante limitação funcional. Farsetti et $a l^{(23)}$ observam que, após 25 anos de seguimento em 14 pacientes tratados clinicamente, não houve alterações estéticas e funcionais em relação à avaliação clínica inicial; concluem que, sempre que há por parte da família e do paciente expectativa de melhora, o tratamento cirúrgico está indicado, preferencialmente utilizando uma técnica que inclua ressecção da parte superior da escápula.

Boon et $a l^{(24)}$ relatam um caso tratado cirurgicamente em que, previamente ao procedimento, foi feita dissecção em 16 cadáveres para melhor conhecimento da anatomia local. Observam que o nervo escapular dorsal, localizado na borda medial superior da escápula, é o que apresenta maior risco de lesão; os nervos acessório e supraescapular raramente estão em risco.

Dendane et $a l^{(25)}$ enfatizam que os candidatos ideais a cirurgias de reposicionamento da escápula são aqueles com menos de quatro anos de idade, enquanto Greitemann et $a l^{(26)}$ consideram que o ideal seria a operação abaixo dos seis anos. Khairouni et al ${ }^{(27)}$ afirmam que tanto a idade do paciente quanto a presença do osso omovertebral não influenciam os resultados. Doita et $a l^{(28)}$ obtiveram bons resultados estéticos em dois pacientes operados na fase adulta, porém utilizaram técnicas apenas de ressecções ósseas, sem rebaixamento da escápula. Ross e Cruess ${ }^{(29)}$ analisaram 77 casos operados e encontraram os melhores resultados com relação ao ganho de elevação com as técnicas de Woodward ${ }^{(8)}$ e Green ${ }^{(17)}$; ambas incluem o rebaixamento da escápula. Zhang et $a l^{(30)}$ não consideram o rebaixamento da escápula para melhora estética como objetivo principal e afirmam que apenas a ressecção da parte súpero-medial da escápula e do osso omovertebral, quando presente, é um seguro e efetivo método de tratamento.

Em apenas dois de nossos pacientes (casos 4 e 7) as indicações cirúrgicas foram com objetivos puramente estéticos. Todos foram operados na primeira década de vida. Por entendermos ser o reposicionamento da escápula fundamental para alcançar melhora funcional, além da estética, optamos por utilizar a técnica de Green com algumas modificações, que foram introduzidas em nosso serviço pelo Dr. Ruy Rocha de Macedo, das quais não encontramos relatos na literatura pesquisada. A principal delas foi a fixação da escápula na crista ilíaca posterior contralateral com fio de aço subcutâneo, em vez de se fazer a tração esquelética. A possibilidade de quebra do fio de aço, que ocorreu em três pacientes, e a necessidade de um segundo procedimento para retirada desse fio podem ser consideradas desvantagens da técnica cirúrgica utilizada. Mas esses fatores não alteraram os resultados finais.

Associado ao procedimento de rebaixamento da escápula, a morcelização (osteotomia) da clavícula, considerada como um primeiro tempo cirúrgico para muitos autores, pode ser realizada em deformidades graves para evitar compressão do plexo braquial ${ }^{(1,2,3,16)}$. Em nossa casuística, esse procedimento não foi realizado, não havendo tal complicação. McMurtry et al ${ }^{(31)}$ consideram que evitar correção excessiva da posição da escápula é a melhor maneira de prevenir complicações neurológicas 
Carson et $a l^{(11)}$ obtiveram ganho de elevação de $29^{\circ} \mathrm{com}$ rebaixamento médio de $1,6 \mathrm{~cm}$, enquanto o de Grogan et $a l^{(32)}$ foi de $37^{\circ} \mathrm{com} 2 \mathrm{~cm}$ de rebaixamento. Ambos utilizaram a técnica de Woodward. Em nossos casos obtivemos ganho médio de $39^{\circ}$ de elevação e $2,7 \mathrm{~cm}$ de rebaixamento.

Wilkinson e Campbell ${ }^{(14)}$ e Cavendish ${ }^{(6)}$ afirmam que a associação da deformidade de Sprengel com alterações esqueléticas graves seria uma contraindicação cirúrgica por não oferecer benefício estético significativo. Apesar do ganho de $40^{\circ}$ de elevação, o paciente (caso 1) que apresentava escoliose grave associada não estava satisfeito no pós-operatório inicial. Porém, no seguimento mais tardio, tanto o paciente como seus familiares se declararam satisfeitos com o resultado da cirurgia.

\section{REFERÊNCIAS}

1. Rockwood CA Jr, Matsen FA 3rd. The Shoulder, 2nd ed., Philadelphia: W.B. Saunders Company; 1998.p.105-9.

2. Tachdjian MO. Orthopedic pediatric. 2th ed. Philadelphia: W.B. Saunders; 1990.

3. Borges JLP, Shah A, Torres BC, Bowen JR. Modified Woodward procedure for Sprengel deformity of the shoulder: long-term results. J Pediatr Orthop. 1996;16: 508-13.

4. Leibovic SJ, Ehrlich MG, Zaleske DJ. Sprengel deformity. J Bone Joint Surg Am. 1990;72:192-7.

5. Balout E, Trouilloud P, Giroux EA, Grammont PM. Ipsilateral omovertebral bones in the levator scapulae muscle and the rhomboid muscle in a Sprengel deformity: case report. Acta Orthop Belgica. 1998;64:92-5.

6. Cavendish ME. Congenital elevation of the scapula. J Bone Joint Surg Br. 1972; 54:395-408.

7. Cho TJ, Choi IH, Chung CY, Hwang JK. The Sprengel deformity: morphometric analysis using 3D-CT and its clinical relevance. J Bone Joint Surg. 2000; 82:711-8.

8. Woodward JW. Congenital elevation of the scapula: correction by release and transplantation of the muscle origin: a preliminary report. J Bone Joint Surg. 1961;43:219-28.

9. Chinn DH. Prenatal ultrasonographic diagnosis of Sprengel's deformity. J Ultrasound Med. 2001; 20(6):693-7.

10. Weinstein SL, Buckwalter JA. Ortopedia de Turek: princípios e sua aplicação, 5a ed. São Paulo: Manole; 2000. p. 400-1.

11. Carson WG, Lovell WW, Whitesides TE. Congenital elevation of the scapula: surgical correction by the Woodward procedure. J Bone Joint Surg Am 1981;63:1199-207.

12. Jeannopoulos CL. Congenital elevation of the scapula. J Bone Joint Surg. Am. 1952; 34:883-92.

13. Mooney JF, White DR, Glazier S. Previously unreported structure associated with Sprengel deformity. J Pediatr Orthop. 2009; 29(1):26-8.

14. Wilkinson JA, Campbell D. Scapular osteotomy for Sprengel's shoulder. J Bone Joint Surg Br. 1980;62:486-90.

15. Mears DC. Partial resection of the scapula and a release of the long head of triceps for the management of Sprengel's Deformity. J Pediatric Orthop. 2001; 21:242-5.

16. ZoppiAF, FerreiraAAF, Robazzi PSM, FerreiraAAN, Benegas E, CostaAJF. Deformidade de Sprengel - Revisão de 15 casos operados. Rev Bras Ortop. 1995;30(9):665-8.
Em relação à estética, houve ganho de pelo menos dois graus segundo a classificação de Cavendish, o que contribuiu para o alto índice de satisfação dos pacientes.

\section{CONCLUSÕES}

A análise dos resultados dos nove pacientes com deformidade de Sprengel operados pela técnica de Green modificada, rebaixando e fixando a escápula à crista ilíaca posterior contralateral com fio de aço, nos mostrou que houve melhora tanto do ponto de vista estético quanto funcional; todos os pacientes e/ou seus familiares ficaram satisfeitos com o tratamento e as complicações relacionadas com a técnica não interferiram no resultado final.
17. Green WT. The surgical correction of congenital elevation of scapula (Sprengel deformity). Proceedings of the American Orthopedic Association. J Bone Joint Surg Am. 1957;(39):1439.

18. Aydinli U, Ozturk C, Akesen B, Ozer O. Surgical treatment of sprengel's deformity: a modified Green procedure. Acta Orthop Belg. 2005;71(3):264-8.

19. Monterrubio CA, Cázares RO. Tratamiento de la deformidad de Sprengel con la técnica Gren modificada/ Treatment of the Sprengel's deformity by a modified Green technique. Rev Mex Ortop Traumatol. 1994;8(6):305-9.

20. Lech O, Reginaldo SS. Deformidade de Sprengel (Escápula Alada). In: Lech O. Defeitos congênitos nos membros superiores. Rio de Janeiro: Medsi; 2003.p.75-83.

21. Van Heat AE. Congenital disorders of the hand and upper extremity. Pediatr Clin North Am. 1996;43-5:1119-21.

22. Sartoris D. Principles of shoulder imaging, New York Mc Graw-Hill, 1995:144-7.

23. Farsetti $P$, Weinstein SL, Caterini R, De Maio F, Ippolito E. Sprengel's deformity: long-term follow-up study of 22 cases. J Pediatr Orthop B. 2003;12(3):202-10.

24. Boon JM, Potgieter D, Van Jaarsveld Z, Frantzen DJM. Congenital undescended scapula (Sprengel deformity): a case study. Clin Anat 2002;15(2):139-42.

25. Dendane AM, Amrani A, El Medhi T, El Alami Z, Gourinda H, Miri A. Congenital elevation of the scapula. Tunis Med. 2004;82(11):1032-7.

26. Greitemann B, Rondhuis JJ, Karbowski A. Treatment of congenital elevation of the scapula: $10(2-18)$ year follow-up of 37 cases of Sprengel's deformity. Acta Orthop Scand. 1993;64(3):365-8.

27. Khairouni A, Bensahel H, Csukonyi Z, Desgrippes Y, Pennecot GF. Congenital high scapula. J Pediatr Orthop B. 2002;1(1):85-8.

28. Doita M, Lio H, Mizuno K. Surgical management of Sprengel's deformity in adults: a report of two cases. Clin Orthop Relat Res. 2000;(371):119-24.

29. Ross DM, Cruess RL. The surgical correction of congenital elevation of the scapula: a review of seventy-seven cases. Clin Orthop Relat Res.1977;(125):17-23.

30. Zhang ZM, Zhang J, Lu ML, Cao GL, Dai LY. Partial scapulectomy for congenital elevation of the scapula. Clin Orthop Relat Res. 2007;(457):171-5.

31. McMurtry I, Bennet GC, Bradish C. Osteotomy for congenital elevation of the scapula (Sprengel's deformity). J Bone Joint Surg Br. 2005;87(7):986-9.

32. Grogan DP, Stanley EA, Bobechko WP. The congenital undescended scapula: surgical correction by the Woodward procedure. J Bone Joint Surg Br.1983; 65(4):598-605. 